\title{
Low Discrepancy Initialized Particle Swarm Optimization for Solving Constrained Optimization Problems
}

\author{
Millie Pant, Radha Thangaraj \\ Department of Paper Technology, IIT Roorkee, Saharanpur, India.
}

\author{
Ajith Abraham* \\ Machine Intelligence Research Labs (MIR Labs)
}

Scientific Network for Innovation and Research Excellence

P.O. Box 2259 Auburn, Washington 98071-2259, USA

ajith.abraham@ieee.org

\begin{abstract}
Population based metaheuristics are commonly used for global optimization problems. These techniques depend largely on the generation of initial population. A good initial population may not only result in a better fitness function value but may also help in faster convergence. Although these techniques have been popular since more than three decades very little research has been done on the initialization of the population. In this paper, we propose a modified Particle Swarm Optimization (PSO) called Improved Constraint Particle Swarm Optimization (ICPSO) algorithm for solving constrained optimization. The proposed ICPSO algorithm is initialized using quasi random Vander Corput sequence and differs from unconstrained PSO algorithm in the phase of updating the position vectors and sorting every generation solutions. The performance of ICPSO algorithm is validated on eighteen constrained benchmark problems. The numerical results show that the proposed algorithm is a quite promising for solving constraint optimization problems.
\end{abstract}

Keywords: Particle Swarm Optimization, Constrained Optimization Problems, Quasi Random, Vander Corput Sequence

\footnotetext{
*Address for correspondence: Machine Intelligence Research Labs (MIR Labs), Scientific Network for Innovation and Research Excellence, P.O. Box 2259 Auburn, Washington 98071-2259, USA.
} 


\section{Introduction}

Most of the problems occurring in the field technology and engineering can be formulated as global optimization problems. Mathematical models of such problems is often complex. The objective function may be multimodal having several local and global optima. Many real-world optimization problems are solved subject to sets of constraints. Such problems are called constrained optimization problems (COP). The search space in COPs consists of two kinds of solutions: feasible and infeasible. Feasible points satisfy all the constraints, while infeasible points violate at least one of them. Therefore, the final solution of an optimization problem must satisfy all constraints. A constrained optimization problem may be distinguished as a Linear Programming Problem (LPP) and Nonlinear Programming Problem (NLP). In this paper we have considered NLP problems where either the objective function or the constraints or both are nonlinear in nature. There are many traditional methods in the literature for solving NLP. However, most of the traditional methods require certain auxiliary properties (like convexity, continuity etc.) of the problem and also most of the traditional techniques are suitable for only a particular type of problem (for example Quadratic Programming Problems, Geometric Programming Problems etc). Keeping in view the limitations of traditional techniques researchers have proposed the use of stochastic optimization methods and intelligent algorithms for solving NLP which may be constrained or unconstrained. Based on the research efforts in literature, constraint handling methods have been categorized in a number of classes $[2,3,4,5]$.

In the past few decades researchers have shown significant interest in population based stochastic search techniques for dealing with global optimization problems. Some popular population based metaheuristics include Genetic Algorithms [6, 7, 8], Ant Colony Optimization [9], Particle Swarm Optimization [10], Differential Evolution [11] etcetera. The first step in all these algorithms is "generate an initial population"; but how the population is to be generated finds little or no mention.

The initial generation of random numbers plays an important role in the population based search techniques for optimization. The uniform distribution of points in the search domain is very likely to improve the performance of a search technique that depends largely on the generation of random number numbers. Now the question arises which method should be used to generate random numbers which will result in faster convergence of the algorithm without compromising with the quality of solution. The most commonly used method for generating the initial population is the use of an inbuilt subroutine available in most of the programming languages for generating random numbers. Though this method is very simple from the programming point of view, it is not very efficient because the computer generated numbers do not cover the search space uniformly. Despite the relevance of distribution of initial population this topic has not gained much attention in the EA community.

In the present study we have made use of quasi random Vander Corput sequence to initialize the population. Some previous instances where low discrepancy sequences have been used to improve the performance of optimization algorithms include [12, 13, 14, 15, 16]. Kimura and Matsumura [12] have used Halton sequence for initializing the Genetic Algorithms (GA) population and have shown that a real coded GA performs much better when initialized with a quasi random sequence in comparison to a GA which initialized with a population having uniform probability distribution. Instances where quasi random sequences have been used for initializing the swarm in PSO can be found in $[13,14,15,16]$. In $[14,15,16]$ authors have made use of Sobol and Faure sequences. Similarly, Nguyen et al. [13] have shown a detailed comparison of Halton Faure and Sobol sequences for initializing the swarm. In the pre- 
vious studies, it has already been shown that the performance of Sobol sequence dominates the performance of Halton and Faure sequences.

The authors suggested the use of quasi random sequences for the initial generation of population in PSO and recoded its performance for unconstrained optimization. In [17], the authors used Vander Corput sequence and Sobol sequence for the initial generation of random numbers in the basic PSO and compared its performance with the PSO in which computer generated random numbers were used for initial population. The numerical results showed that the proposed quasi random sequences significantly improve the performance of basic PSO. Encouraged by the versions made for solving unconstrained optimization problems in [17], in this paper the authors propose the use of quasi random Vander Corput sequence for solving constrained optimization problems.

This paper presents an Improved Constraint Particle Swarm Optimization (ICPSO) algorithm for solving constrained optimization problems. Its initial population is generated using Vander Corput sequence and its constraint solving approach is similar to the approach used by K. Zielinski et al. [18] where a personal or neighborhood best solution $\bar{g}$ is substituted by a new solution $\bar{x}$ if:

- Both vectors are feasible, but $\bar{x}$ yield the smaller objective function value.

- $\bar{x}$ is feasible and $\bar{g}$ is not.

- Both vectors are infeasible, but $\bar{x}$ results in the lower sum of constraint violations.

The proposed ICPSO is different from the algorithm proposed in [18], as the swarm is initialized by Low discrepancy Vander Corput Sequence and above rules are applied during the updating of position vectors. In the present study we have concentrated our work on PSO which is relatively a new member to a class of population based search technique. To the best of our knowledge, no results are available on the performance of low discrepancy sequence for solving constrained optimization problems. Moreover, the proposed concept may be applied to any of the population based search technique for solving constrained optimization problems.

The structure of the paper is as follows: in Section 2, we give a brief definition of low discrepancy sequences and Vander Corput sequence. In Section 3, we explain the Particle Swarm Optimization Algorithm, in Section 4, the proposed ICPSO algorithm is given. Section 5 deals with experimental settings and test problems, Section 6 gives the numerical results and discussion and finally the paper conclude with Section 7.

\section{Quasi Random Vander Corput Sequence}

\subsection{Low discrepancy or Quasi Random Sequences}

The most common practice of generating random numbers is the one using an inbuilt subroutine (available in most of the programming languages), which uses a uniform probability distribution to generate random numbers. This method is not very proficient as it has been shown that uniform pseudorandom number sequences have discrepancy of order $(\log (\log N))^{1 / 2}$ and thus do not achieve the lowest possible discrepancy. Subsequently, researchers have proposed an alternative way of generating 'quasirandom' numbers through the use of low discrepancy sequences. Their discrepancies have been shown to be optimal, of order $(\log N)^{s} / N$ [19], [20]. Quasirandom sequences, on the other hand are more useful for global optimization, because of the variation of random numbers that are produced in each iteration. 
Many of the relevant low discrepancy sequences are linked to the Van der Corput sequence introduced initially for dimension $s=1$ and base $b=2$ [21]. The Van der Corput discovery inspired other quasi random sequences like Halton [22], Faure, Sobol [23, 24], etc. However, it has been reported that Halton and Faure sequences do not work too well when the search space has large dimensions. Keeping this fact in mind we decided to scrutinize the performance of PSO using Van der Corput sequence along with Sobol sequence (which is said be superior than other low discrepancy sequences according to the previous studies) for swarm initialization and tested them for solving global optimization problems in large dimension search spaces.

\subsection{Van der Corput Sequence}

A Van der Corput sequence is a low-discrepancy sequence over the unit interval first published in 1935 by the Dutch mathematician J. G. Van der Corput. It is a digital $(0,1)$-sequence, which exists for all bases $b \geq 2$. It is defined by the radical inverse function $\varphi_{b}: N_{0} \rightarrow[0,1)$. If $n \in N_{0}$ has the $b$-adic expansion

$$
n=\sum_{j=0}^{T} a_{j} b^{j-1}
$$

with $a_{j} \in\{0, \ldots, b-1\}$, and $T=\left\lfloor\log _{b} n\right\rfloor$ then $\varphi_{b}$ is defined as

$$
\varphi_{b}(n)=\sum_{j=0}^{T} \frac{a_{j}}{b^{j}}
$$

In other words, the $j$ th $b$-adic digit of $n$ becomes the $j$ th $b$-adic digit of $\varphi_{b}(n)$ behind the decimal point. The Van der Corput sequence in base $b$ is then defined as $\left(\varphi_{b}(n)\right)_{n \geq 0}$.

The elements of the Van der Corput sequence (in any base) form a dense set in the unit interval: for any real number in $[0,1]$ there exists a sub sequence of the Van der Corput sequence that converges towards that number. They are also uniformly distributed over the unit interval. Figures 2 and 3 depict the initial 500 points generated by using the inbuilt subroutine and by using Vander Corput sequence respectively. The figures clearly show that the initial points generated by using quasi random sequences cover the search space more evenly in comparison to the pseudo random numbers generated by using computer subroutine. The distribution of sample points in space using computer generated pseudo random numbers and sample points generated using quasi random Vander Corput sequence are shown in Fig 1 and 2 respectively. From these figures it can be easily seen that the sample points generated by quasi random sequence are far more uniformly distributed in comparison to the computer generated pseudo random sample points generated by using inbuilt subroutine.

\section{Particle Swarm Optimization Algorithm}

Particle Swarm Optimization (PSO) is a relatively newer addition to a class of population based search technique for solving numerical optimization problems. Its mechanism is inspired from the complex social behavior shown by the natural species like flock of birds, school of fish and even crowd of human beings. The particles or members of the swarm fly through a multidimensional search space looking for 

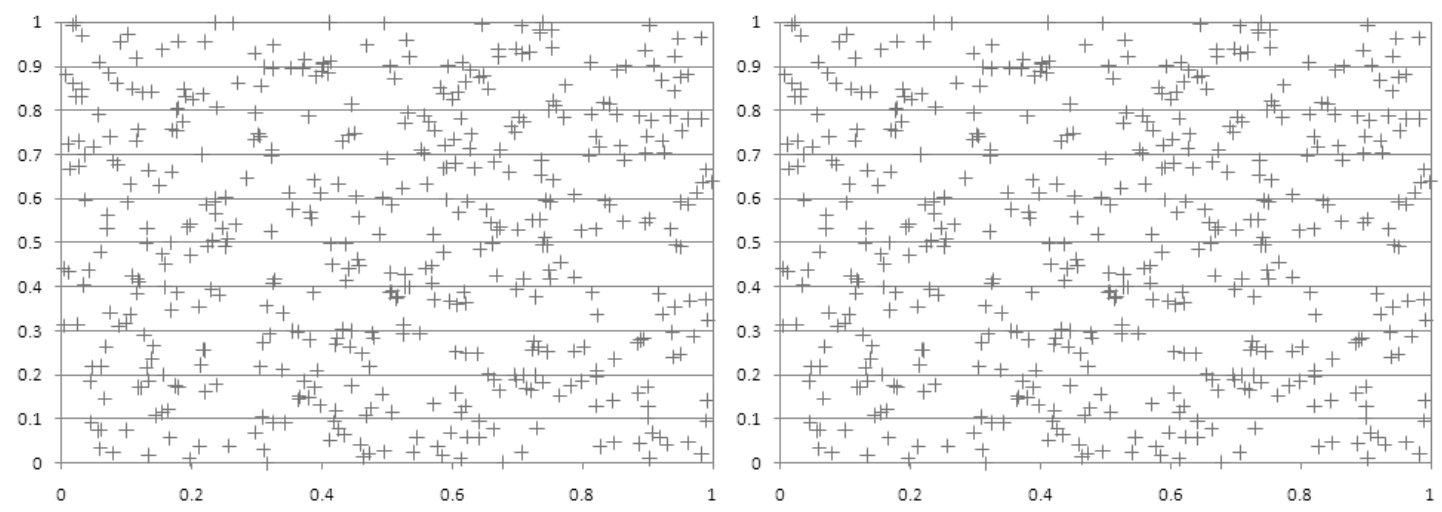

Figure 1. Sample points generated using: Fig.1 a pseudo random sequence, Fig.2 Vander Corput sequence

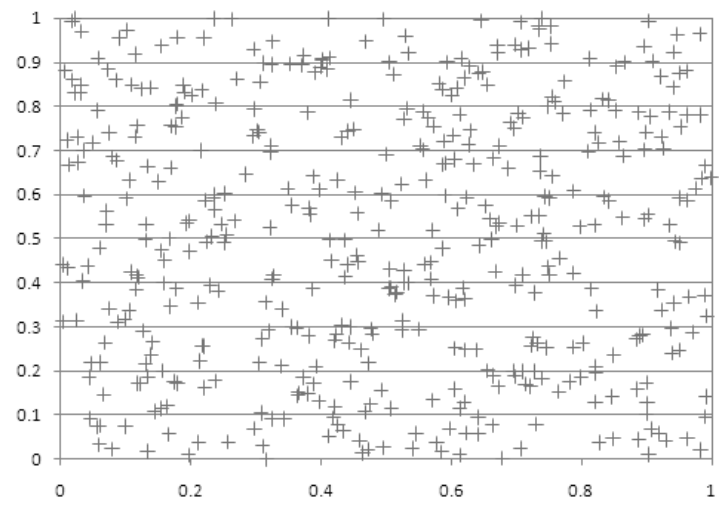

Figure 2. Sample points generated using a pseudo random sequence

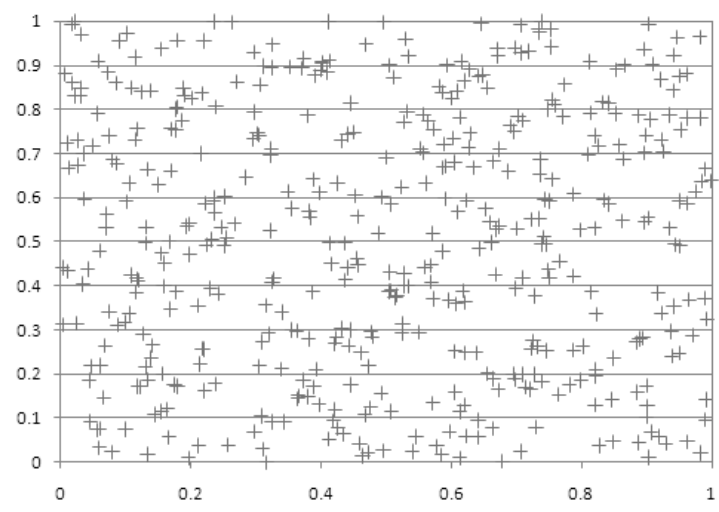

Figure 3. Sample points generated using Vander Corput sequence 
a potential solution. Each particle adjusts its position in the search space from time to time as per its own experience and also as per the position of its neighbors (or colleagues).

For a $D$-dimensional search space the position of the $i$ th particle is represented as $X_{i}=\left(x_{i 1}, x_{i 2}, \ldots\right.$, $\left.x_{i D}\right)$. Each particle maintains a memory of its previous best position $P_{i}=\left(p_{i 1}, p_{i 2}, \ldots, p_{i D}\right)$ and a velocity $V_{i}=\left(v_{i 1}, v_{i 2}, \ldots, v_{i D}\right)$ along each dimension. At each iteration, the $P$ vector of the particle with best fitness in the local neighborhood, designated $g$, and the $P$ vector of the current particle are combined to adjust the velocity along each dimension and a new position of the particle is determined using that velocity. The two basic equations which govern the working of PSO are that of velocity vector and position vector are given by:

$$
\begin{aligned}
v_{i d} & =\omega v_{i d}+c_{1} r_{1}\left(p_{i d}-x_{i d}\right)+c_{2} r_{2}\left(p_{g d}-x_{i d}\right) \\
x_{i d} & =x_{i d}+v_{i d}
\end{aligned}
$$

The first part of equation (3) represents the inertia of the previous velocity, the second part is the cognition part and it tells us about the personal thinking of the particle, the third part represents the cooperation among particles and is therefore named as the social component [25]. Acceleration constants $c_{1}, c_{2}$ [26] and inertia weight $\omega$ [27] are predefined by the user and $r_{1}, r_{2}$ are the uniformly generated random numbers in the range of $[0,1]$.

\section{Proposed ICPSO Algorithm}

The proposed algorithm ICPSO is a simple algorithm for solving constraint optimization problems, it is easy to implement. It is initialized using Vander Corput sequence and it differs from unconstrained PSO in the phase of updating the position vectors and sorting every generation solutions. The proposed ICPSO algorithm uses the low discrepancy Vander Corput Sequence for initializing the population and uses the following three selection criteria after calculating the new particle

1. If the new particle and the previous particle are feasible then select the best one

2. If both the particles are infeasible then select the one having smaller constraint violation

3. If one is feasible and the other one is infeasible then select the feasible one.

Also at the end of every iteration, the particles are sorted by using the three criteria:

1. Sort feasible solutions in front of infeasible solutions

2. Sort feasible solutions according to their fitness function values

3. Sort infeasible solutions according to their constraint violations.

The computational steps of ICPSO algorithm is given Algorithm 4:

\section{Benchmark problems, Experimental Settings}

\subsection{Benchmark problems}

The general NLP is given by nonlinear objective function $f$, which is to be minimized/maximized with respect to the design variables $\bar{x}=\left(x_{1}, x_{2}, \ldots, x_{n}\right)$ and the nonlinear inequality and equality constraints. The mathematical models of the problems considered in the paper are of the type: 


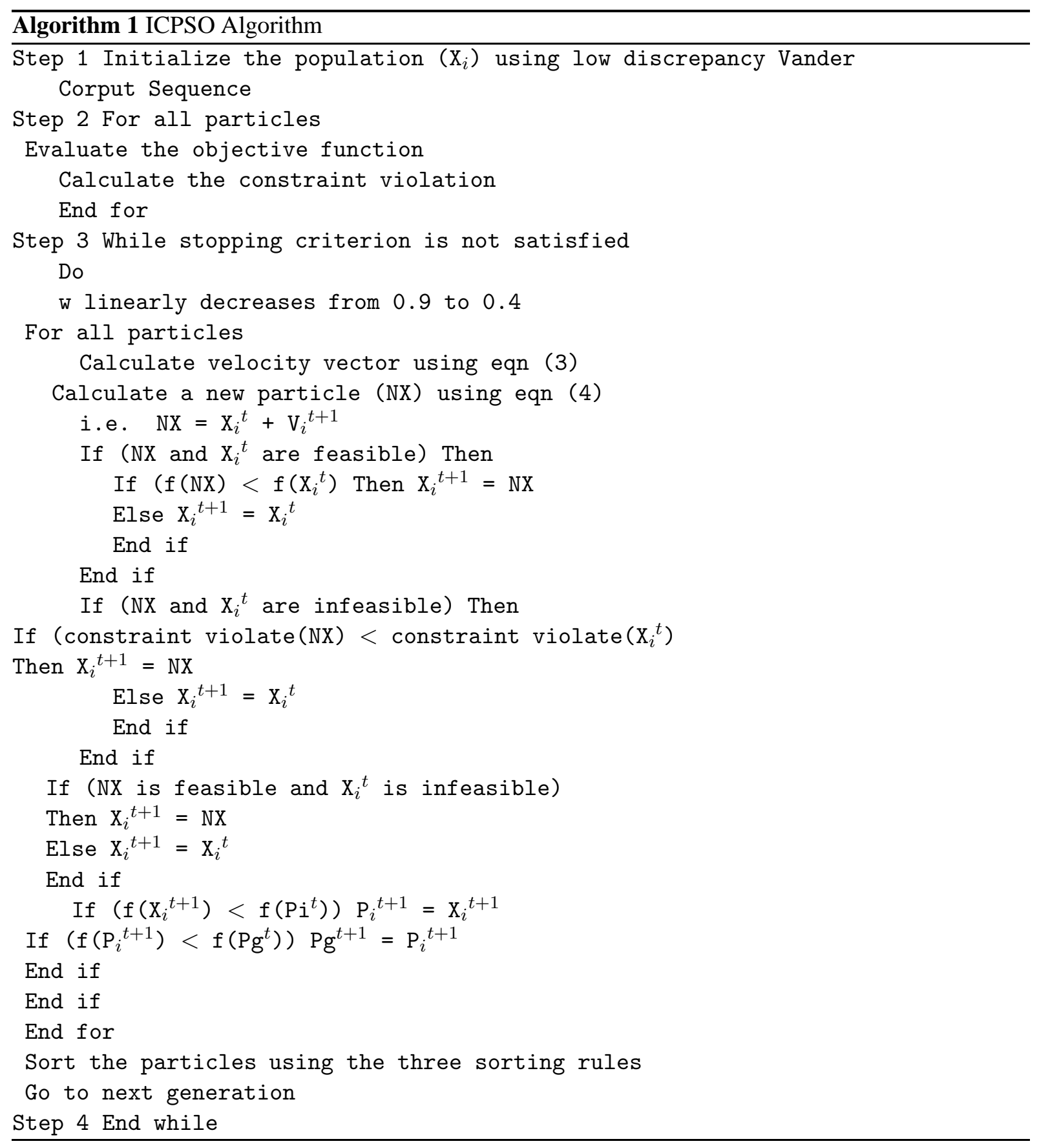


Minimize/Maximize $f(\bar{x})$

Subject to:

$$
\begin{aligned}
g_{j}(\bar{x}) & \leq 0, j=1, \ldots, p \\
h_{k}(\bar{x}) & =0, k=1, \ldots, q
\end{aligned}
$$

$x_{i \min } \leq x_{i} \leq x_{i \max }(i=1, \ldots, n)$, where $p$ and $q$ are the number of inequality and equality constraints respectively.

A set of 18 constrained benchmark problems is considered to evaluate the performance of the proposed ICPSO. All the problems are nonlinear in nature i.e. either the objective function or the constraints or both have a nonlinear term in it. The mathematical models of the problems along with the optimal solution are given in Appendix A.

\subsection{Experimental settings}

A total of 25 runs for each experimental setting are conducted and the average fitness of the best solutions throughout the run is recorded. The population size is taken as 50 . A linearly decreasing inertia weight is used which starts at 0.9 and ends at 0.4 , with the user defined parameters $c_{1}=c_{2}=2.0$ and $r_{1}, r_{2}$ as uniformly distributed random numbers between 0 and 1 . The proposed ICPSO algorithm is compared with two more variations of PSO.

\section{Comparison Criteria, Results and Discussion}

\subsection{Comparison Criteria}

We used several criteria to measure the performance of the proposed ICPSO algorithm and to compare it with other versions of PSO. In Tables 1 - 4, we recorded the performance the proposed ICPSO in terms of best worst and average fitness function value along with the standard deviation (Std) while increasing the NFE (number of function evaluations) to three different values $5 \times 10^{3}, 5 \times 10^{4}, 5 \times 10^{5}$.

In Tables 5 and 6, the performance of ICPSO is compared with two other variants of PSO for solving constrained optimization problems. The comparison criteria for all the algorithms taken in the present study are given as:

Feasible Run: A run during which at least one feasible solution is found in Max NFE.

Successful Run: A run during which the algorithm finds a feasible solution $x$ satisfying $\left(f(x)-f\left(x^{*}\right)\right) \leq 0.0001$.

Feasible Rate $=(\#$ of feasible runs $) /$ total runs

Success Rate $=(\#$ of successful runs $) /$ total runs

Success Performance $=$ mean $($ FEs for successful runs $) \times x(\#$ of total runs $) /(\#$ of successful runs $)$

\subsection{Results and Discussion}

From Tables $1-4$, we can see that the performance of the proposed ICPSO improves with the increase in the number of function evaluations. This is quite an expected out come. However it can be seen that $5 \times 10^{4}$ NFE is sufficient for reaching a good optimum solution which lies in the vicinity of the true 
Table 1. Fitness function values achieved when $N F E=5 \times 10^{3}, N F E=5 \times 10^{4}$ and $N F E=5 \times 10^{5}$ for problems f01 - f05

\begin{tabular}{|l|l|l|l|l|l|l|}
\hline FES & & $\mathrm{f} 01$ & $\mathrm{f} 02$ & $\mathrm{f} 03$ & $\mathrm{f} 04$ & $\mathrm{f} 05$ \\
\hline \multirow{5}{*}{$5 \times 10^{3}$} & Best & -12.7810 & 0.412234 & -0.5123 & -30665.5314 & 5126.2298 \\
\cline { 2 - 7 } & Worst & -10.3994 & 0.354648 & -0.2144 & -30665.3480 & 5189.3433 \\
\cline { 2 - 7 } & Mean & -11.3257 & 0.363072 & -0.4231 & -30665.3712 & 5165.7069 \\
\cline { 2 - 7 } & Std & 0.77603 & 0.021727 & 0.0393 & 0.228162 & 56.177 \\
\hline \multirow{5}{*}{$5 \times 10^{4}$} & Best & -15 & 0.803138 & -0.7181 & -30665.5386 & 5126.4967 \\
\cline { 2 - 7 } & Worst & -15 & 0.784856 & -0.3990 & -30665.5386 & 5126.4967 \\
\cline { 2 - 7 } & Mean & -15 & 0.793258 & -0.6495 & -30665.5386 & 5126.4967 \\
\cline { 2 - 7 } & Std & $9.3 \mathrm{e}-09$ & 0.00976 & 0.1294 & $1.02 \mathrm{e}-12$ & $5.53 \mathrm{e}-05$ \\
\hline \multirow{5}{*}{$5 \times 10^{5}$} & Best & -15 & 0.803618 & -0.8324 & -30665.5386 & 5126.4967 \\
\cline { 2 - 7 } & Worst & -15 & 0.794661 & -0.4751 & -30665.5386 & 5126.4967 \\
\cline { 2 - 7 } & Mean & -15 & 0.803113 & -0.7563 & -30665.5386 & 5126.4967 \\
\cline { 2 - 7 } & Std & $1.5 \mathrm{e}-11$ & 0.009781 & 0.0245 & 0.0000 & $2.40 \mathrm{e}-12$ \\
\hline
\end{tabular}

Table 2. Fitness function values achieved when $N F E=5 \times 10^{3}, N F E=5 \times 10^{4}$ and $N F E=5 \times 10^{5}$ for problems f06 - $\mathrm{f} 10$

\begin{tabular}{|r|r|r|r|r|r|r|}
\hline NFE & & f06 & f07 & f08 & f09 & f10 \\
\hline \multirow{4}{*}{$5 \times 10^{3}$} & Best & -6961.8127 & 25.5805 & -0.095826 & 680.6481 & 8207.3551 \\
\cline { 2 - 7 } & Worst & -6939.9306 & 28.9778 & -0.095826 & 681.1337 & 8399.2033 \\
\cline { 2 - 7 } & Mean & -6958.7191 & 27.6499 & -0.095826 & 680.7835 & 8344.4623 \\
\cline { 2 - 7 } & Std & 10.1347 & 0.9488 & $2.77 \mathrm{e}-18$ & 0.1498 & 2.734 \\
\hline \multirow{5}{*}{$5 \times 10^{4}$} & Best & -6961.8138 & 24.3062 & -0.095826 & 680.6303 & 7049.2533 \\
\cline { 2 - 7 } & Worst & -6961.8138 & 24.3118 & -0.095826 & 681.0767 & 7049.2738 \\
\cline { 2 - 7 } & Mean & -6961.8138 & 24.4006 & -0.095826 & 680.6683 & 7049.2697 \\
\cline { 2 - 7 } & Std & $9.09 \mathrm{e}-13$ & 0.01911 & $4.80 \mathrm{e}-19$ & 0.089227 & 0.01456 \\
\hline \multirow{5}{*}{$5 \times 10^{5}$} & Best & -6961.8138 & 24.3062 & -0.095826 & 680.6301 & 7049.2480 \\
\cline { 2 - 7 } & Worst & -6961.8138 & 24.3246 & -0.095826 & 680.6435 & 7049.2480 \\
\cline { 2 - 7 } & Mean & -6961.8138 & 24.3073 & -0.095826 & 680.6329 & 7049.2480 \\
\cline { 2 - 7 } & Std & $1.98 \mathrm{e}-15$ & 0.00402 & 0.0000 & 0.0525 & $1.81 \mathrm{e}-13$ \\
\hline
\end{tabular}


Table 3. Fitness function values achieved when $N F E=5 \times 10^{3}, N F E=5 \times 10^{4}$ and $N F E=5 \times 10^{5}$ for problems f11 - f15

\begin{tabular}{|l|l|l|l|l|l|l|}
\hline NFE & & $\mathrm{f} 11$ & $\mathrm{f} 12$ & $\mathrm{f} 13$ & $\mathrm{f} 14$ & $\mathrm{f} 15$ \\
\hline \multirow{4}{*}{$5 \times 10^{3}$} & Best & 0.7499 & -1 & 0.4923 & -44.4379 & 961.7302 \\
\cline { 2 - 7 } & Worst & 0.8539 & -1 & 0.9997 & -39.8987 & 962.0497 \\
\cline { 2 - 7 } & Mean & 0.8102 & -1 & 0.8807 & -42.1293 & 961.7565 \\
\cline { 2 - 7 } & Std & 0.0733 & 0.0000 & 0.1940 & 1.3022 & 1.9369 \\
\hline \multirow{5}{*}{$5 \times 10^{4}$} & Best & 0.7499 & -1 & 0.3212 & -47.6380 & 961.7150 \\
\cline { 2 - 7 } & Worst & 0.7499 & -1 & 0.6389 & -45.7222 & 962.6006 \\
\cline { 2 - 7 } & Mean & 0.7499 & -1 & 0.4783 & -46.2218 & 962.2491 \\
\cline { 2 - 7 } & Std & $2.22 \mathrm{e}-16$ & 0.0000 & 0.1067 & 1.0495 & 0.8847 \\
\hline \multirow{5}{*}{$5 \times 10^{5}$} & Best & 0.7499 & -1 & 0.0531 & -47.7648 & 961.7150 \\
\cline { 2 - 7 } & Worst & 0.7499 & -1 & 0.434 & -47.7648 & 961.7150 \\
\cline { 2 - 7 } & Mean & 0.7499 & -1 & 0.32736 & -47.7648 & 961.7150 \\
\cline { 2 - 7 } & Std & $2.22 \mathrm{e}-16$ & 0.0000 & 0.171017 & $4.71 \mathrm{e}-15$ & $4.42 \mathrm{e}-13$ \\
\hline
\end{tabular}

Table 4. Fitness function values achieved when $N F E=5 \times 10^{3}, N F E=5 \times 10^{4}$ and $N F E=5 \times 10^{5}$ for problems $\mathrm{f} 16-\mathrm{f} 18$

\begin{tabular}{|l|l|l|l|l|}
\hline NFE & & $\mathrm{f} 16$ & $\mathrm{f} 17$ & $\mathrm{f} 18$ \\
\hline \multirow{4}{*}{$5 \times 10^{3}$} & Best & -1.9015 & 8967.5800 & -0.6485 \\
\cline { 2 - 5 } & Worst & -1.8991 & 11028.714 & -0.4833 \\
\cline { 2 - 5 } & Mean & -1.9001 & 9311.915 & -0.5261 \\
\cline { 2 - 5 } & Std & 0.00251 & 7.618 & 0.05928 \\
\hline \multirow{4}{*}{$5 \times 10^{4}$} & Best & -1.9051 & 8868.7455 & -0.8657 \\
\cline { 2 - 5 } & Worst & -1.9051 & 10903.986 & -0.8644 \\
\cline { 2 - 5 } & Mean & -1.9051 & 9070.5204 & -0.8650 \\
\cline { 2 - 5 } & Std & $7.90 \mathrm{e}-16$ & 4.8995 & 0.00066 \\
\hline \multirow{4}{*}{$5 \times 10^{5}$} & Best & -1.9051 & 8853.5338 & -0.8660 \\
\cline { 2 - 5 } & Worst & -1.9051 & 8853.5338 & -0.8660 \\
\cline { 2 - 5 } & Mean & -1.9051 & 8853.5338 & -0.8660 \\
\cline { 2 - 5 } & Std & $8.05 \mathrm{e}-17$ & $1.00 \mathrm{e}-12$ & $1.06 \mathrm{e}-16$ \\
\hline
\end{tabular}


Table 5. Comparison Results: NFE to achieve the fixed accuracy level $\left(\left(f(x)-f\left(x^{*}\right)\right) \leq 0.0001\right)$, success rate, Feasible Rate and Success Performance for problems f01 - f10

\begin{tabular}{|c|c|c|c|c|c|c|c|}
\hline Problem & Algo & Best & Worst & Mean & $\begin{array}{l}\text { Feasible } \\
\text { Rate }(\%)\end{array}$ & $\begin{array}{l}\text { Success } \\
\text { Rate }(\%)\end{array}$ & $\begin{array}{l}\text { Success } \\
\text { Perf. }\end{array}$ \\
\hline \multirow{3}{*}{ f01 } & ICPSO & 25250 & 55250 & 29796 & 100 & 100 & 29796 \\
\hline & [17] & 25273 & 346801 & 76195 & 100 & 52 & 146530 \\
\hline & [27] & 95100 & 106900 & 101532 & 100 & 100 & 101532 \\
\hline \multirow{3}{*}{$\mathrm{f} 02$} & ICPSO & 81800 & 135750 & 115850 & 100 & 100 & 115850 \\
\hline & [17] & - & - & - & 100 & 0 & - \\
\hline & [27] & 180000 & 327900 & 231193 & 100 & 56 & 412844.3878 \\
\hline \multirow{3}{*}{ f03 } & ICPSO & - & - & - & 100 & 0 & - \\
\hline & [17] & - & - & - & 100 & 0 & - \\
\hline & [27] & 450100 & 454000 & 450644 & 100 & 100 & 450644 \\
\hline \multirow{3}{*}{ f04 } & ICPSO & 7750 & 12650 & 9568 & 100 & 100 & 9568 \\
\hline & [17] & 15363 & 25776 & 20546 & 100 & 100 & 20546 \\
\hline & [27] & 74300 & 85000 & 79876 & 100 & 100 & 79876 \\
\hline \multirow{3}{*}{ f05 } & ICPSO & 13350 & 65400 & 19286 & 100 & 100 & 19286 \\
\hline & [17] & 94156 & 482411 & 364218 & 100 & 16 & 2276363 \\
\hline & [27] & 450100 & 457200 & 452256 & 100 & 100 & 452256 \\
\hline \multirow{3}{*}{ f06 } & ICPSO & 7300 & 9600 & 8252 & 100 & 100 & 8252 \\
\hline & [17] & 16794 & 22274 & 20043 & 100 & 100 & 20043 \\
\hline & [27] & 47800 & 61100 & 56508 & 100 & 100 & 56508 \\
\hline \multirow{3}{*}{ f07 } & ICPSO & 29050 & 57800 & 40046 & 100 & 100 & 40046 \\
\hline & [17] & 315906 & 338659 & 327283 & 100 & 8 & 4091031 \\
\hline & [27] & 198600 & 444100 & 352592 & 100 & 96 & 367282.9861 \\
\hline \multirow{3}{*}{ f08 } & ICPSO & 1050 & 1350 & 1158 & 100 & 100 & 1158 \\
\hline & [17] & 1395 & 3921 & 2360 & 100 & 100 & 2360 \\
\hline & [27] & 2800 & 8400 & 6124 & 100 & 100 & 6124 \\
\hline \multirow{3}{*}{ f09 } & ICPSO & 10450 & 29550 & 16248 & 100 & 100 & 16248 \\
\hline & [17] & 45342 & 84152 & 58129 & 100 & 100 & 58129 \\
\hline & [27] & 77000 & 129000 & 97544 & 100 & 100 & 97544 \\
\hline \multirow{3}{*}{ f010 } & ICPSO & 66050 & 84900 & 75920 & 100 & 100 & 75920 \\
\hline & [17] & 290367 & 486655 & 426560 & 100 & 32 & 1332999 \\
\hline & [27] & 398000 & 475600 & 452575 & 100 & 16 & 2828593.75 \\
\hline
\end{tabular}


Table 6. Comparison Results: NFE to achieve the fixed accuracy level $\left(\left(f(x)-f\left(x^{*}\right)\right) \leq 0.0001\right)$, success rate, Feasible Rate and Success Performance for problems f11 - f18

\begin{tabular}{|c|c|c|c|c|c|c|c|}
\hline $\begin{array}{l}\text { Problem } \\
\text { Problem }\end{array}$ & $\begin{array}{l}\text { Algo } \\
\text { Algo }\end{array}$ & $\begin{array}{l}\text { Best } \\
\text { Best }\end{array}$ & $\begin{array}{l}\text { Worst } \\
\text { Worst }\end{array}$ & $\begin{array}{l}\text { Mean } \\
\text { Mean }\end{array}$ & $\begin{array}{l}\text { Feasible } \\
\text { Rate (\%) }\end{array}$ & $\begin{array}{l}\text { Success } \\
\text { Rate (\%) }\end{array}$ & $\begin{array}{l}\text { Success } \\
\text { Perf. }\end{array}$ \\
\hline \multirow{3}{*}{ f11 } & ICPSO & 1650 & 24250 & 13630 & 100 & 100 & 13630 \\
\hline & {$[17]$} & 5475 & 21795 & 16386 & 100 & 100 & 16386 \\
\hline & [27] & 450100 & 450100 & 450100 & 100 & 100 & 450100 \\
\hline \multirow{3}{*}{ f12 } & ICPSO & 850 & 1100 & 976 & 100 & 100 & 976 \\
\hline & [17] & 1409 & 9289 & 4893 & 100 & 100 & 4893 \\
\hline & [27] & 3300 & 10900 & 8088 & 100 & 100 & 8088 \\
\hline \multirow{3}{*}{ f13 } & ICPSO & 88700 & 111100 & 102512 & 100 & 16 & 640700 \\
\hline & [17] & - & - & - & 100 & 0 & - \\
\hline & [27] & 450100 & 453200 & 450420 & 100 & 100 & 450420 \\
\hline \multirow{3}{*}{ f14 } & ICPSO & 21250 & 339550 & 50614 & 100 & 100 & 50614 \\
\hline & [17] & - & - & - & 100 & 0 & - \\
\hline & [27] & - & - & - & 100 & 0 & - \\
\hline \multirow{3}{*}{ f15 } & ICPSO & 7400 & 128100 & 54306 & 100 & 100 & 54306 \\
\hline & [17] & 17857 & 348138 & 176827 & 100 & 80 & 221033 \\
\hline & [27] & 450100 & 450100 & 450100 & 100 & 100 & 450100 \\
\hline \multirow{3}{*}{ f16 } & ICPSO & 7100 & 10650 & 8732 & 100 & 100 & 8732 \\
\hline & [17] & 24907 & 51924 & 33335 & 100 & 100 & 33335 \\
\hline & [27] & 43400 & 53900 & 49040 & 100 & 100 & 49040 \\
\hline \multirow{3}{*}{ f17 } & ICPSO & 256800 & 463350 & 408506 & 96 & 72 & 567369 \\
\hline & [17] & - & - & - & 100 & 0 & - \\
\hline & [27] & - & - & - & 100 & 0 & - \\
\hline \multirow{3}{*}{ f18 } & ICPSO & 53600 & 89900 & 71694 & 100 & 100 & 71694 \\
\hline & [17] & 85571 & 455907 & 191220 & 100 & 80 & 239026 \\
\hline & [27] & 120800 & 394900 & 214322 & 100 & 92 & 232958.4121 \\
\hline
\end{tabular}

optimum value, under the present parameter settings. Also, we can see that except for problem number 5 (g05), where the standard deviation (std) is 56.177, the std for all the remaining problems is quite low. This shows the consistency of the proposed ICPSO algorithm. The superior performance of ICPSO is more visible from Tables 5 and 6 where the results are recorded after fixing the accuracy at 0.0001 . In these tables we can see that the proposed ICPSO gave a better or at par performance with the other two algorithms. We will now take the comparison criteria one-by-one and discuss them briefly. The first criterion is that of a feasible run. A run is said to be feasible if at least one feasible solution is obtained in maximum number of function evaluations. According to this criterion all the algorithm gave $100 \%$ feasible rate for all the test problems except ICPSO which gave $96 \%$ feasible rate for test problem f17. However, if we observe the second criterion which is of successful run and is recorded when the algorithm finds a feasible solution satisfying the given accuracy $(=0.0001)$ it can be seen that the proposed ICPSO outperforms the other algorithms in all the test cases including f17. In f17, the 
percentage of success rate for ICPSO is 72 , whereas the other algorithms were not able to reach the prescribed accuracy in any of the run. The third criterion is that of the success performance which depends on the feasibility rate and success rate, as described in the previous subsection. Here also ICPSO gave a better performance in comparison to the other two algorithms taken for comparison.

\section{Conclusion}

In the present study a low discrepancy Vander Corput sequence initialized particle swarm optimization called ICPSO is proposed for solving constrained optimization. Besides the initialization process, the proposed algorithm differs from the basic PSO in the updating of position vectors and sorting of every generation solutions. The proposed technique for solving constrained optimization problems, though used for PSO in this paper, can be applied to any other population based search technique with minor modifications. The empirical analysis of the proposed ICPSO algorithm on 18 constrained benchmark problems and its comparison with other algorithms show that the proposed algorithm is quite promising for solving constrained problems. In the present study we have used the Vander Corput sequence however any other low discrepancy sequences like Sobol or Halton may also be used to initialize the population. We are continuing the further study of the algorithm and are using it for solving constrained real life problems taken from various fields of Science and Engineering.

\section{References}

[1] K. Deb, A. Pratap, S. Agarwal, and T. Meyarivan, "A fast and elitist multiobjective genetic algorithm: NSGAII”, IEEE Transactions on Evolutionary Computation, vol. 6, no. 2, 2002.

[2] A. P. Engelbrecht, "Fundamentals of Computational Swarm Intelligence", England : John Wiley \& Sons Ltd., 2005.

[3] G. Coath, S. K. Halgamuge, "A Comparison of Constraint- Handling Methods for the Application of Particle Swarm Optimization to Constrained Nonlinear Optimization Problems", In Proc. of the IEEE Congress on Evolutionary Computation, Vol. 4, pp. 2419 - 2425, 2003.

[4] S. Koziel, Z. Michalewicz, "Evolutionary Algorithms, Homomorphus Mappings, and Constrained Optimization”, Evolutionary Computation, Vol. 7(1), pp. 19 - 44, 1999.

[5] Z. Michalewicz, "A Survey of Constraint Handling Techniques in Evolutionary Computation Methods”, In Proc. of the Fourth Annual Conf. on Evolutionary Programming, pp. 135 - 155, 1995.

[6] Y. Li, M.Gen, "Non-linear mixed integer programming problems using genetic algorithm and penalty function”, In Proc. of 1996 IEEE Int. Conf. on SMC, pp. 2677 - 2682, 1996.

[7] Y. Takao, M. Gen, T. Takeaki, Y. Li, "A method for interval 0-1 number non-linear programming problems using genetic algorithm”, Computers and Industrial Engineering, Vol. 29, pp. 531 - 535, 1995.

[8] J. F. Tang, D.Wang, et al., "A hybrid genetic algorithm for a type of nonlinear programming problem", Computer Math. Applic, Vol. 36(5), pp. 11 - 21, 1998.

[9] M. Dorigo, V. Maniezzo, A. Colori, “Ant system optimization by a colony of cooperating agents", IEEE Trans. on system, Man, and Cybernetics, Vol. 26(1), pp. 28 - 41, 1996.

[10] J. Kennedy, R. Eberhart, "Particle Swarm Optimization”, IEEE International Conference on Neural Networks (Perth, Australia), IEEE Service Center, Piscataway, NJ, IV: pp. 1942-1948, 1995. 
[11] R. Storn and K. Price, "Differential Evolution - a simple and efficient Heuristic for global optimization over continuous spaces", Journal Global Optimization. 11, 1997, pp. 341 - 359.

[12] S. Kimura and K. Matsumura, "Genetic Algorithms using low discrepancy sequences", in proc of GEECO 2005, pp. 1341 - 1346.

[13] Nguyen X. H., Nguyen Q. Uy., R. I. Mckay and P. M. Tuan, "Initializing PSO with Randomized LowDiscrepancy Sequences: The Comparative Results", In Proc. of IEEE Congress on Evolutionary Algorithms, 2007, pp. 1985 - 1992.

[14] K.E. Parsopoulos and M.N. Vrahatis, "Particle Swarm Optimization in noisy and continuously changing environments", in Proceedings of International Conference on Artificial Intelligence and soft computing, 2002, pp. 289-294.

[15] R. Brits and A.P. Engelbrecht and F. van den Bergh, "A niching Particle Swarm Optimizater", In proceedings of the fourth Asia Pacific Conference on Simulated Evolution and learning, 2002, pp 692 - 696.

[16] R. Brits and A.P. Engelbrecht and F. van den Bergh, "Solving systems of unconstrained Equations using Particle Swarm Optimization", In proceedings of the IEEE Conference on Systems, Man and Cybernetics, Vol. 3pp. 102 - 107, 2002.

[17] Millie Pant, Radha Thangaraj and Ajith Abraham "Improved Particle Swarm Optimization with Lowdiscrepancy Sequences", IEEE Cong. on Evolutionary Computation (CEC’08), Hong Kong, 2008.

[18] Karin Zielinski and Rainer Laur : "Constrained Single-Objective Optimization Using Particle Swarm Optimization", IEEE Congress on Evolutionary Computation, Sheraton Vancouver Wall Centre Hotel, Vancouver, BC, Canada July 16-21, 2006, pp. 443 - 450

[19] E. J. Gentle, "RandomNumber Generation and Monte Carlo Methods", Springer-Verlog, 1998.

[20] D. E. Knuth, "The Art of Computer Programming", Semi numerical Algorithms, Vol. 2, Addison-Wesley, 1998.

[21] J. G. van der Corput, Verteilungsfunktionen. Proc. Ned. Akad. v. Wet., 38: pp. 813-821, 1935.

[22] J. Halton, "On the efficiency of certain quasi-random sequences of points in evaluating multi-dimensional integrals," Numerische Mathematik, Vol. 2, 1960, pp. 84 - 90.

[23] I. M. Sobol', "On the distribution of points in a cube and the approximate evaluation of integrals," USSR Computational Mathematics and Mathematical Physics, Vol. 7, 1967, pp. 86 - 112.

[24] I. M. Sobol', "Uniformly distributed sequences with an additional uniform property," USSR Computational Mathematics and Mathematical Physics, Vol. 16, 1976, pp. 236 - 242.

[25] J. Kennedy, "The Particle Swarm: Social Adaptation of Knowledge", IEEE International Conference on Evolutionary Computation (Indianapolis, Indiana), IEEE Service Center, Piscataway, NJ, 1997, pp. 303 - 308.

[26] R. C. Eberhart and Y. Shi, "Particle Swarm Optimization: developments, Applications and Resources", IEEE Int. Conference on Evolutionary Computation, 2001, pp. 81 - 86.

[27] Y. H. Shi and R. C. Eberhart, "A Modified Particle Swarm Optimizer", IEEE International Conference on Evolutionary Computation, Anchorage, Alaska, 1998, pp. 69 - 73.

[28] Angel E. Mu?noz-Zavala, Arturo Hern?andez-Aguirre, Enrique R. Villa-Diharce and Salvador BotelloRionda: "PESO+ for Constrained Optimization" IEEE Congress on Evolutionary Computation Sheraton Vancouver Wall Centre Hotel, Vancouver, BC, Canada July 16-21, 2006, pp. 231 - 238. 


\section{A. Appendix}

1. F01:

Minimize $f(x)=5 \sum_{i=1}^{4} x_{i}-5 \sum_{i=1}^{4} x_{i}^{2}-\sum_{i=5}^{13} x_{i}$

Subject to:

$$
\begin{aligned}
& g_{1}(x)=2 x_{1}+2 x_{2}+x_{10}+x_{11}-10 \leq 0 \\
& g_{2}(x)=2 x_{1}+2 x_{3}+x_{10}+x_{12}-10 \leq 0 \\
& g_{3}(x)=2 x_{2}+2 x_{3}+x_{11}+x_{12}-10 \leq 0 \\
& g_{4}(x)=-8 x_{1}+x_{10} \leq 0 \\
& g_{5}(x)=-8 x_{2}+x_{11} \leq 0 \\
& g_{6}(x)=-8 x_{3}+x_{12} \leq 0 \\
& g_{7}(x)=-2 x_{4}-x_{5}+x_{10} \leq 0 \\
& g_{8}(x)=-2 x_{6}-x_{7}+x_{11} \leq 0 \\
& g_{9}(x)=-2 x_{8}-x_{9}+x_{12} \leq 0 \\
& 0 \leq x_{i} \leq 1(i=1,2, \ldots, 9), 0 \leq x_{i} \leq 100,(i=10,11,12) 0 \leq x_{13} \leq 1
\end{aligned}
$$

The optimum value is $f\left(x^{*}\right)=-15$ at $x^{*}=(1,1,1,1,1,1,1,1,1,3,3,3,1)$

Constraints $g_{1}, g_{2}, g_{3}, g_{7}, g_{8}, g_{9}$ are active.

2. F02:

Maximize

$$
f(x)=\left|\frac{\sum_{i=1}^{n} \cos ^{4}\left(x_{i}\right)-2 \prod_{i=1}^{n} \cos ^{2}\left(x_{i}\right)}{\sum_{i=1}^{n} i x_{i}^{2}}\right|
$$

Subject to:

$$
\begin{gathered}
g_{1}(x)=0.75-\prod_{i=1}^{n} x_{i} \leq 0 \\
g_{2}(x)=\sum_{i=1}^{n} x_{i}-7.5 n \leq 0 \\
0 \leq x_{i} \leq 10(i=1,2, \ldots, n), n=20
\end{gathered}
$$

The optimum value is unknown. The known best value is $f\left(x^{*}\right)=0.803619$ Constraint $g_{1}$ is active.

3. F03:

Minimize

$$
f(x)=-(\sqrt{n})^{n} \prod_{i=1}^{n} x_{i}
$$


Subject to:

$$
\begin{gathered}
h_{1}(x)=\sum_{i=1}^{n} x_{i}^{2}-1=0 \\
0 \leq x_{i} \leq 10(i=1,2, \ldots, n)
\end{gathered}
$$

The optimum value is $f\left(x^{*}\right)=-1$ at $x^{*}=(1 / \sqrt{n}), n=10$.

4. F04

Minimize $f(x)=5.3578547 x_{3}^{2}+0.8356891 x_{1} x_{5}+37.293239 x_{1}-40792.141$

Subject to:

$$
\begin{aligned}
& g_{1}(x)=85.334407+0.0056858 x_{2} x_{5}+0.0006262 x_{1} x_{4}-0.0022053 x_{3} x_{5} \\
& g_{2}(x)=80.51249+0.0071317 x_{2} x_{5}+0.0029955 x_{1} x_{2}+0.0021813 x_{3}^{2} \\
& g_{3}(x)=9.300961+0.0047026 x_{3} x_{5}+0.0012547 x_{1} x_{3}+0.0019085 x_{3} x_{4}
\end{aligned}
$$

$$
\begin{gathered}
0 \leq g_{1}(x) \leq 92 \\
90 \leq g_{2}(x) \leq 110 \\
20 \leq g_{3}(x) \leq 25
\end{gathered}
$$

$78 \leq x_{1} \leq 102,33 \leq x_{2} \leq 45,27 \leq x_{i} \leq 45(i=3,4,5)$.

The optimum value is $f\left(x^{*}\right)=-30665.539$ at $x^{*}=(78,33,29.995256025682,45,36.775812905788)$

5. F05

Minimize $f(x)=3 x_{1}+0.000001 x_{1}^{3}+2 x_{2}+(0.000002 / 3) x_{2}^{3}$

Subject to:

$$
\begin{aligned}
g_{1}(x) & =-x_{4}+x_{3}-0.55 \leq 0 \\
g_{2}(x) & =-x_{3}+x_{4}-0.55 \leq 0 \\
h_{3}(x) & =1000 \sin \left(-x_{3}-0.25\right)+1000 \sin \left(-x_{4}-0.25\right)+894.8-x_{1}=0 \\
h_{4}(x) & =1000 \sin \left(x_{3}-0.25\right)+1000 \sin \left(x_{3}-x_{4}-0.25\right)+894.8-x_{2}=0 \\
h_{5}(x) & =1000 \sin \left(x_{4}-0.25\right)+1000 \sin \left(x_{4}-x_{3}-0.25\right)+1294.8=0 \\
0 \leq x_{i} \leq 1200 & (i=1,2),-0.55 \leq x_{2} \leq 0.55(i=3,4) .
\end{aligned}
$$

The optimum value is $f\left(x^{*}\right)=5126.4981$ at $x^{*}=(679.9463,1026.067,0.1188764,-0.3962336)$.

6. F06

Minimize $f(x)=\left(x_{1}-10\right)^{3}+\left(x_{2}-20\right)^{3}$ 
Subject to:

$$
\begin{aligned}
& g_{1}(x)=-\left(x_{1}-5\right)^{2}-\left(x_{2}-5\right)^{2}+100 \leq 0 \\
& g_{2}(x)=\left(x_{1}-6\right)^{2}+\left(x_{2}-5\right)^{2}-82.81 \leq 0
\end{aligned}
$$

$13 \leq x_{1} \leq 100,0 \leq x_{2} \leq 100$

The optimum value is $f\left(x^{*}\right)=-6961.81388$ at $x^{*}=(14.095,0.84296)$.

7. F07:

Minimize $f(x)=x_{1}^{2}+x_{2}^{2}+x_{1} x_{2}-14 x_{1}-16 x_{2}+\left(x_{3}-10\right)^{2}+4\left(x_{4}-5\right)^{2}+\left(x_{5}-5\right)^{2}$ $+2\left(x_{6}-1\right)^{2}+5 x_{7}^{2}+7\left(x_{8}-11\right)^{2}+2\left(x_{9}-10\right)^{2}+\left(x_{10}-7\right)^{2}+45$

Subject to:

$g_{1}(x)=-105+4 x_{1}+5 x_{2}-3 x_{7}+9 x_{8} \leq 0 g_{2}(x)=10 x_{1}-8 x_{2}-17 x_{7}+2 x_{8} \leq 0 g_{3}(x)=$ $-8 x_{1}+2 x_{2}+5 x_{9}-2 x_{10}-12 \leq 0 g_{4}(x)=3\left(x_{1}-2\right)^{2}+4\left(x_{2}-3\right)^{2}+2 x_{3}^{2}-7 x_{4}-120 \leq 0 g_{5}(x)=$ $5 x_{1}^{2}+8 x_{2}+\left(x_{3}-6\right)^{2}-2 x_{4}-40 \leq 0 g_{6}(x)=x_{1}^{2}+2\left(x_{2}-2\right)^{2}-2 x_{1} x_{2}+14 x_{5}-6 x_{6} \leq 0 g_{7}(x)=$ $0.5\left(x_{1}-8\right)^{2}+2\left(x_{2}-4\right)^{2}+3 x_{5}^{2}-x_{6}-30 \leq 0 g_{8}(x)=-3 x_{1}+6 x_{2}+12\left(x_{9}-8\right)^{2}-7 x_{10} \leq$ $0-10 \leq x_{i} \leq 10(i=1,2, \ldots, 10)$

The optimum value is $f\left(x^{*}\right)=24.3062091$ at $x^{*}=(2.171996,2.363683,8.773926,5.095984$, $0.9906548,1.430574,1.321644,9.828726,8.280092,8.375927)$

Constraints $\mathrm{g}_{1}, \mathrm{~g}_{2}, \mathrm{~g}_{3}, \mathrm{~g}_{4}, \mathrm{~g}_{5}$ and $\mathrm{g}_{6}$ are active.

8. F08:

Maximize $f(x)=\frac{\sin ^{3}\left(2 \pi x_{1}\right) \sin \left(2 \pi x_{2}\right)}{x_{1}^{3}\left(x_{1}+x_{2}\right)}$

Subject to:

$g_{1}(x)=x_{1}^{2}-x_{2}+1 \leq 0 g_{2}(x)=1-x_{1}+\left(x_{2}-4\right)^{2} \leq 0$

$0 \leq x_{i} \leq 10(i=1,2)$.

The optimum value is $f\left(x^{*}\right)=0.095825$ at $x^{*}=(1.2279713,4.2453733)$.

9. F09:

Minimize $f(x)=\left(x_{1}-10\right)^{2}+5\left(x_{2}-12\right)^{2}+x_{3}^{4}+3\left(x_{4}-11\right)^{2}+10 x_{5}^{6}+7 x_{6}^{2}+x_{7}^{4}-4 x_{6} x_{7}-10 x_{6}-8 x_{7}$ Subject to:

$g_{1}(x)=-127+2 x_{1}^{2}+3 x_{2}^{4}+x_{3}+4 x_{4}^{2}+5 x_{5} \leq 0 g_{2}(x)=-282+7 x_{1}+3 x_{2}+10 x_{3}^{2}+x_{4}-x_{5} \leq$ $0 g_{3}(x)=-196+23 x_{1}+x_{2}^{2}+6 x_{6}^{2}-8 x_{7} \leq 0 g_{4}(x)=4 x_{1}^{2}+x_{2}^{2}-3 x_{1} x_{2}+2 x_{3}^{2}+5 x_{6}-11 x_{7} \leq$ $0-10 \leq x_{i} \leq 10(i=1,2, \ldots, 7)$

The optimum value is $f\left(x^{*}\right)=680.6300573$ at $x^{*}=(2.330499,1.951372,-0.4775414,4.365726$, $-0.624487,1.038131,1.5942270)$. 
10. F10:

Minimize $f(x)=x_{1}+x_{2}+x_{3}$

Subject to:

$g_{1}(x)=-1+0.0025\left(x_{4}+x_{6}\right) \leq 0 g_{2}(x)=-1+0.0025\left(x_{5}+x_{7}-x_{4}\right) \leq 0 g_{3}(x)=-1+$ $0.01\left(x_{8}-x_{5}\right) \leq 0 g_{4}(x)=-x_{1} x_{6}+833.33252 x_{4}+100 x 1-83333.333 \leq 0 g_{5}(x)=-x_{2} x_{7}+$ $1250 x_{5}+x_{2} x_{4}-1250 x_{4} \leq 0 g_{6}(x)=-x_{3} x_{8}+1250000+x_{3} x_{5}-2500 x_{5} \leq 0$

$-100 \leq x_{1} \leq 10000,1000 \leq x_{i} \leq 10000(i=2,3), 10 \leq x_{i} \leq 1000(i=4, \ldots, 8)$.

The optimum value is $f\left(x^{*}\right)=7049.25$ at

$x^{*}=(579.19,1360.13,5109.5979,182.0174,295.5985,217.9799,286.40,395.5979)$.

11. F11:

Minimize $f(x)=x_{1}^{2}+\left(x_{2}-1\right)^{2}$

Subject to:

$h_{1}(x)=x_{2}-x_{1}^{2}=0-1 \leq x_{i} \leq 1(i=1,2)$

The optimum value is $f\left(x^{*}\right)=0.75$ at $x^{*}=( \pm 1 / \sqrt{2}, 1 / 2)$.

12. F12:

Minimize $f(x)=-\left(100-\left(x_{1}-5\right)^{2}-\left(x_{2}-5\right)^{2}-\left(x_{3}-5\right)^{2}\right) / 100$

Subject to:

$g(x)=\left(x_{1}-p\right)^{2}+\left(x_{2}-q\right)^{2}+\left(x_{3}-r\right)^{2}-0.0625 \leq 0$

$0 \leq x_{i} \leq 10, i=1,2,3, p, q, r=1,2, \ldots, 9$

The optimum value is $f\left(x^{*}\right)=-1$ at $x^{*}=(5,5,5)$.

13. F13:

Minimize $f(x)=e^{x_{1} x_{2} x_{3} x_{4} x_{5}}$

Subject to:

$h_{1}(x)=x_{1}^{2}+x_{2}^{2}+x_{3}^{2}+x_{4}^{2}+x_{5}^{2}-10=0 h_{2}(x)=x_{2} x_{3}-5 x_{4} x_{5}=0 h_{3}(x)=x_{1}^{3}+x_{2}^{3}+1=0$

$-2.3 \leq x_{i} \leq 2.3, i=1,2$

$-3.2 \leq x_{i} \leq 3.2, i=3,4,5$

The optimum value is $f\left(x^{*}\right)=0.0539$ at $x^{*}=(-1.7171,1.5957,1.8272,-0.7636,-0.7636)$.

14. F14:

Minimize $f(x)=\sum_{i=1}^{10} x_{i}\left(c_{i}+\ln \frac{x_{i}}{\sum_{j=1}^{10} x_{j}}\right)$

Subject to:

$h_{1}(x)=x_{1}+2 x_{2}+2 x_{3}+x_{6}+x_{10}-2=0 h_{2}(x)=x_{4}+2 x_{5}+x_{6}+x_{7}-1=0 h_{3}(x)=$ $x_{3}+x_{7}+x_{8}+2 x_{9}+x_{10}-1=0$ 
$0<x_{i} \leq 10, i=1, \ldots, 10$

Where $c_{1}=-6.089, c_{2}=-17.164, c_{3}=-34.054, c_{4}=-5.914, c_{5}=-24.721, c_{6}=-14.986$, $c_{7}=-24.1, c_{8}=-10.708, c_{9}=-26.662, c_{10}=-22.179$

The optimum value is $f\left(x^{*}\right)=-47.7648$ at $x^{*}=(0.04066,0.14772,0.78320,0.00141,0.48529$, $0.00069,0.02740,0.017950,0.03732,0.09688)$

\section{F15:}

Minimize $f(x)=1000-x_{1}^{2}-2 x_{2}^{2}-x_{3}^{2}-x_{1} x_{2}-x_{1} x_{3}$

Subject to:

$h_{1}(x)=x_{1}^{2}+x_{2}^{2}+x_{3}^{2}-25=0 h_{2}(x)=8 x_{1}+14 x_{2}+7 x_{3}-56=0$

$0 \leq x_{i} \leq 10, i=1,2,3$

The optimum value is $f\left(x^{*}\right)=961.7150$ at $x^{*}=(3.5121,0.2169,3.5521)$

16. F16:

Minimize $f(x)=0.00011 y_{14}+0.1365+0.00002358 y_{13}+0.000001502 y_{16}+0.0321 y_{12}+$ $0.004324 y_{5}+0.0001 \frac{c_{15}}{c_{16}}+37.48 \frac{y_{2}}{c_{12}}-0.0000005843 y_{17}$

Subject to:

$$
\begin{aligned}
& g_{1}=\frac{0.28}{0.72} y_{5}-y_{4} \leq 0, g_{2}=x_{3}-1.5 x_{2} \leq 0, \\
& g_{3}=3496 \frac{y_{2}}{c_{12}}-21 \leq 0, g_{4}=110.6+y_{1}-\frac{62,212}{c_{17}} \leq 0, g_{5}=y_{1}-405.23 \leq 0, g_{6}=213.1-y_{1} \leq 0 \\
& g_{7}=y_{2}-1053.6667 \leq 0, g_{8}=17.505-y_{2} \leq 0 \\
& g_{9}=y_{3}-35.03 \leq 0, g_{10}=11.275-y_{3} \leq 0, g_{11}=y_{4}-665.585 \leq 0, g_{12}=214.228-y_{4} \leq 0, \\
& g_{13}=y_{5}-584.463 \leq 0, g_{14}=7.458-y_{5} \leq 0, \\
& g_{15}=y_{6}-265.916 \leq 0, g_{16}=0.961-y_{6} \leq 0, g_{17}=y_{7}-7.046 \leq 0, g_{18}=1.612-y_{7} \leq 0, \\
& g_{19}=y_{8}-0.222 \leq 0, g_{20}=0.146-y_{8} \leq 0, g_{21}=y_{9}-273.366 \leq 0, g_{22}=107.99-y_{9} \leq 0, \\
& g_{23}=y_{10}-1286.105 \leq 0, g_{24}=922.693-y_{10} \leq 0, g_{25}=y_{11}-1444.046 \leq 0, g_{26}= \\
& 926.832-y_{11} \leq 0, g_{27}=y_{12}-537.141 \leq 0, g_{28}=18.766-y_{12} \leq 0, g_{29}=y_{13}-3247.039 \leq 0, \\
& g_{30}=1072.163-y_{13} \leq 0, g_{31}=y_{14}-26844.086 \leq 0, \\
& g_{32}=8961.448-y_{14} \leq 0, g_{33}=y_{15}-0.386 \leq 0, g_{34}=0.063-y_{15} \leq 0, g_{35}=y_{16}- \\
& 140,000 \leq 0, \\
& g_{36}=71,084.33-y_{16} \leq 0, g_{37}=y_{17}-12,146,108 \leq 0, g_{38}=2,802,713-y_{17} \leq 0, \\
& 704.4148 \leq x_{1} \leq 906.3855,68.6 \leq x_{2} \leq 288.88,0 \leq x_{3} \leq 134.75,193 \leq x_{4} \leq 287.0966, \\
& 25 \leq x_{5} \leq 84.1988 .
\end{aligned}
$$

Calculations:

$$
\begin{aligned}
& y_{1}=x_{2}+x_{3}+41.6, c_{1}=0.024 x_{4}-4.62, y_{2}=\frac{12.5}{c_{1}}+12, \\
& c_{2}=0.0003535 x_{1}^{2}+0.5311 x_{1}+0.08705 y_{2} x_{1}, c_{3}=0.052 x_{1}+78+0.002377 y_{2} x_{1}, \\
& y_{3}=\frac{c_{2}}{c_{3}}, y_{4}=19 y_{3}, c_{4}=0.04782\left(x_{1}-y_{3}\right)+\frac{0.1956\left(x_{1}-y_{3}\right)^{2}}{x_{2}}+0.6376 y_{4}+1.594 y_{3}, \\
& c_{5}=100 x_{2}, c_{6}=x_{1}-y_{3}-y_{4}, c_{7}=0.95-\frac{c_{4}}{c_{5}}, y_{5}=c_{6} c_{7}, y_{6}=x_{1}-y_{5}-y_{4}-y_{3}
\end{aligned}
$$




$$
\begin{aligned}
& c_{8}=\left(y_{5}+y_{4}\right) 0.995, y_{7}=\frac{c_{8}}{y_{1}}, y_{8}=\frac{c_{8}}{3798}, c_{9}=y_{7}-\frac{0.0663 y_{7}}{y_{8}}-0.3153 \\
& y_{9}=\frac{96.82}{c_{9}}+0.321 y_{1}, y_{10}=1.29 y_{5}+1.258 y_{4}+2.29 y_{3}+1.71 y_{6}, y_{11}=1.71 x_{1}-0.452 y_{4}+0.58 y_{3} \\
& c_{10}=\frac{12.3}{752.3}, c_{11}=\left(1.75 y_{2}\right)\left(0.995 x_{1}\right), c_{12}=0.995 y_{10}+1998, y_{12}=c_{10} x_{1}+\frac{c_{11}}{c_{12}} \\
& y_{13}=c_{12}-1.75 y_{2}, y_{14}=3623+64.4 x_{2}+58.4 x_{3}+\frac{146312}{y_{9}+x_{5}} \\
& c_{13}=0.995 y_{10}+60.8 x_{2}+48 x_{4}-0.1121 y_{14}-5095, y_{15}=\frac{y_{13}}{c_{13}} \\
& y_{16}=148000-331000 y_{15}+40 y_{13}-61 y_{15} y_{13}, c_{14}=2324 y_{10}-28740000 y_{2} \\
& y_{17}=14130,000-1328 y_{10}-531 y_{11}+\frac{c_{14}}{c_{12}}, c_{15}=\frac{y_{13}}{y_{15}}-\frac{y_{13}}{0.52} \\
& c_{16}=1.104-0.72 y_{15}, c_{17}=y_{9}+x_{5} .
\end{aligned}
$$

17. F17:

Minimize $f(x)=f_{1}\left(x_{1}\right)+f_{2}\left(x_{2}\right)$

Constraints:

$$
\begin{aligned}
& f_{1}\left(x_{1}\right)=\left\{\begin{array}{ll}
31 x_{1} & 0 \leq x_{1} \leq 300 \\
30 x_{1} & 300 \leq x_{1} \leq 400
\end{array} f_{2}\left(x_{2}\right)= \begin{cases}28 x_{2} & 0 \leq x_{2} \leq 100 \\
29 x_{2} & 100 \leq x_{2} \leq 200 \\
30 x_{2} & 200 \leq x_{2} \leq 1000\end{cases} \right. \\
& x_{1}=300-\frac{x_{3} x_{4}}{131.078} \cos \left(1.48477-x_{6}\right)+\frac{0.90798 x_{3}^{2}}{131.078} \cos (1.47588) \\
& x_{2}=-\frac{x_{3} x_{4}}{131.078} \cos \left(1.48477+x_{6}\right)+\frac{0.90798 x_{4}^{2}}{131.078} \cos (1.47588) \\
& x_{5}=-\frac{x_{3} x_{4}}{131.078} \sin \left(1.48477+x_{6}\right)+\frac{0.90798 x_{4}^{2}}{131.078} \sin (1.47588) 200-\frac{x_{3} x_{4}}{131.078} \sin \left(1.48477-x_{6}\right)+ \\
& \quad+\frac{0.90079 x_{3}^{2}}{131.078} \sin (1.47588)=0
\end{aligned}
$$

$$
\begin{gathered}
0 \leq x_{1} \leq 400 \\
0 \leq x_{2} \leq 1000 \\
340 \leq x_{3} \leq 420 \\
340 \leq x_{4} \leq 420 \\
-1000 \leq x_{5} \leq 1000 \\
0 \leq x_{6} \leq 0.5236
\end{gathered}
$$

18. F18:

Minimize $f(x)=-0.5\left(x_{1} x_{4}-x_{2} x_{3}+x_{3} x_{9}-x_{5} x_{9}+x_{5} x_{8}-x_{6} x_{7}\right)$

Subject to:

$$
\begin{gathered}
g_{1}(x)=x_{3}^{2}+x_{4}^{2}-1 \leq 0 g_{2}(x)=x_{9}^{2}-1 \leq 0 g_{3}(x)=x_{5}^{2}+x_{6}^{2}-1 \leq 0 g_{4}(x)=x_{1}^{2}+\left(x_{2}-x_{9}\right)^{2}-1 \leq \\
0 g_{5}(x)=\left(x_{1}-x_{5}\right)^{2}+\left(x_{2}-x_{6}\right)^{2}-1 \leq 0 g_{6}(x)=\left(x_{1}-x_{7}\right)^{2}+\left(x_{2}-x_{8}\right)^{2}-1 \leq 0 g_{7}(x)= \\
\left(x_{3}-x_{5}\right)^{2}+\left(x_{4}-x_{6}\right)^{2}-1 \leq 0 \\
g_{8}(x)=\left(x_{3}-x_{7}\right)^{2}+\left(x_{4}-x_{8}\right)^{2}-1 \leq 0
\end{gathered}
$$




$$
\begin{gathered}
g_{9}(x)=x_{7}^{2}+\left(x_{8}-x_{9}\right)^{2}-1 \leq 0 \\
g_{10}(x)=x_{2} x_{3}-x_{1} x_{5} \leq 0 \\
g_{11}(x)=-x_{3} x_{9} \leq 0 \\
g_{12}(x)=x_{5} x_{9} \leq 0 \\
g_{13}(x)=x_{6} x_{7}-x_{5} x_{8} \leq 0
\end{gathered}
$$

$-10 \leq x_{i} \leq 10, i=1, \ldots, 8,0 \leq x_{9} \leq 10$

The optimum value is $f\left(x^{*}\right)=-0.8660$ at $x^{*}=(-0.65777,-0.15341,0.32341,-0.94625$, $-0.65777,-0.75321,0.32341,-0.34646,0.59979)$ 